\title{
Inferior vena cava leiomyosarcoma in an octogenerian
}

\author{
Sudharsan Madhavan, Sameer P Junnarkar, Nicholas Wee Chong Koh, and Vishalkumar G Shelat
}

\author{
Department of General Surgery, Tan Tock Seng Hospital, Singapore
}

\begin{abstract}
Inferior Vena Cava (IVC) leiomyosarcoma (LM) is a rare malignancy of mesenchymal origin with an incidence of $1 / 100,000$. We present an 82-year-old lady with a past history of open cholecystectomy who presented with a large indeterminate mass on abdominal imaging. Open resection of the mass was performed and histology with immunohistochemical staining revealed leiomyosarcoma. She received adjuvant radiotherapy and remained disease free 4 years after. (Ann Hepatobiliary Pancreat Surg 2019;23:274-277)
\end{abstract}

Key Words: Inferior vena cava; Leiomyosarcoma; Octogenerian; Sarcoma; Vascular tumour

\section{INTRODUCTION}

Vascular leiomyosarcomas (LM) constitute around 1-2\% of all soft tissue sarcomas. ${ }^{1}$ Amongst those, LM originating from the Inferior Vena Cava (IVC) is even rarer with incidence of $<1$ in 100,000 adult malignancies. ${ }^{2-4}$ With increased reporting of these cases in recent times, ${ }^{5}$ greater light has been shed on prognosis, survival determinants and characteristics of this peculiar neoplasm. However, there is much ambivalence about the optimal treatment for patients with IVC LM. Radical resection with or without vascular reconstruction, chemotherapy, radiotherapy and chemo-radiotherapy are all potential options but are all widely debated. We describe a case of IVC LM in an octogenarian managed by the hepatobiliary service and patient remained disease free at four years follow up.

\section{CASE}

An 80-year-old Indian lady presented with loss of weight of $5 \mathrm{~kg}$ over 4 months with loss of appetite. There was no history of jaundice or abdominal pain. She has a past medical history of a benign thyroid lesion treated with right hemi-thyroidectomy as well as cholecystitis treated by open cholecystectomy more than 10 years ago. She al- so had ischemic heart disease, diabetes mellitus and hyperlipidemia; all medically controlled. Physical examination was unremarkable. Liver function test, coagulation screen, renal function test, thyroid function test, alpha-fetoprotein, carcinoembryonic antigen and carbohydrate antigen 19-9 were normal. She had colonoscopy and gastroscopy performed one year ago to evaluate anemia and they revealed findings suggestive of hyperplastic rectal polyps and chronic gastritis respectively. Her current haemoglobin level was $10.2 \mathrm{~g} / \mathrm{dl}$. Computed Tomography (CT) scan of the abdomen and pelvis demonstrated a $7.8 \times 6.4 \times 6.2 \mathrm{~cm}$ mass closely abutting the inferior vena cava with no intervening fat plane and within the gallbladder bed (Fig. 1). The mass shows internal calcification and compresses the common bile duct causing proximal biliary dilatation. Mass was separate from liver and head of pancreas and abuts second part of duodenum. Staging CT scan of thorax was normal. Multi-disciplinary tumour board recommended surgical resection for this indeterminate solid tumour.

Intraoperatively, the findings were that of an encapsulated and lobulated mass displacing the common bile duct anteriorly and portal vein medially. The mass was not arising from the duodenum, stomach or pancreas. Trucut biopsy of the mass was sent for frozen section and it re-

Received: July 10, 2018; Revised: November 7, 2018; Accepted: November 15, 2018

Corresponding author: Vishalkumar G Shelat

Department of General Surgery, Tan Tock Seng Hospital, 11 Jalan Tan Tock Seng, 308433, Singapore

Tel: +65-63577807, Fax: +65-63577809, E-mail: vishal_g_shelat@ttsh.com.sg

Copyright (C) 2019 by The Korean Association of Hepato-Biliary-Pancreatic Surgery

This is an Open Access article distributed under the terms of the Creative Commons Attribution Non-Commercial License (http://creativecommons.org/ licenses/by-nc/4.0) which permits unrestricted non-commercial use, distribution, and reproduction in any medium, provided the original work is properly cited. Annals of Hepato-Biliary-Pancreatic Surgery • pISSN: 2508-5778 - eISSN: 2508-5859 
vealed a spindle cell lesion. During dissection it appeared that mass was adherent to inferior vena cava and this needed en bloc resection of anterolateral wall of inferior vena cava. Inadvertent tear of the remnant cystic duct encountered due to adhesions resulting from prior cholecystectomy and this was repaired. Post-operatively, patient was transfused one pint of packed red blood cells

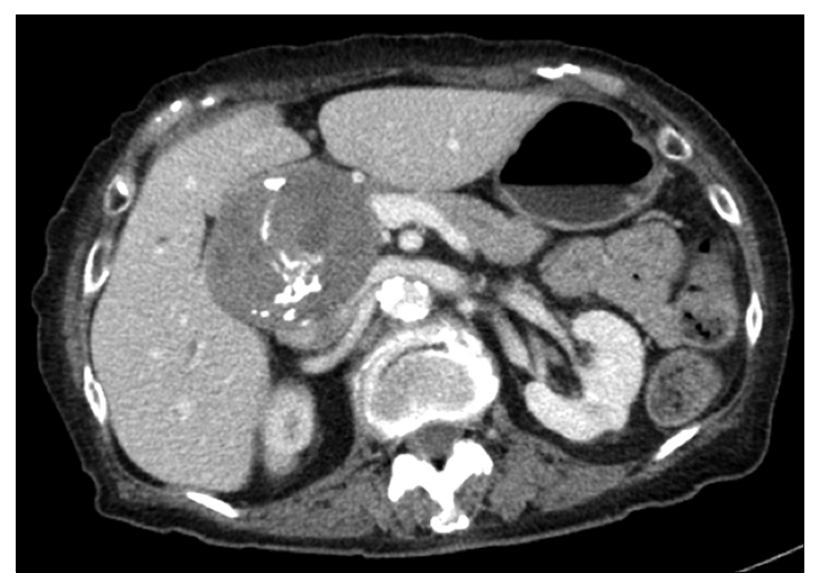

Fig. 1. Preoperative computed tomography of the abdomen and pelvis. Computed tomography scan showing mass with internal calcification which abuts inferior vena cava and is within the gallbladder bed. and discharged on day seven with no complications.

The specimen consisted of a pear-shaped mass, measuring $9.5 \times 5 \times 5 \mathrm{~cm}$ and weighing $157.6 \mathrm{gm}$. Cut sections of the specimen showed a solid mass with a homogenous tan whitish appearance (Fig. 2A). Microscopic examination showed a tumour comprised of intersecting fascicles of spindle cells (Fig. 2B). The tumour cells generally featured elongated 'cigar-shaped' nuclei with abundant eosinophilic cytoplasm; in areas, the tumour cells showed significant nuclear atypia, appearing enlarged, irregular and hyperchromatic (Fig. 2C). No definite areas of necrosis were found. Rare mitotic figures were identified with $>1 / 50$ HPF and the Ki-67 proliferation index was estimated to be around $15 \%$. Immunohistochemical stains showed diffuse positivity within the tumour cells for the smooth muscle markers smooth muscle actin (SMA) and desmin (Fig. 2D), as well as CD34. CD117, DOG-1, S-100 and cytokeratin AE $1 / 3$ were negative. The tumour involved the resection margin.

Multi-disciplinary tumour board recommended adjuvant radiotherapy. 54 Gy of intensity modulated radiation therapy (IMRT) was administered in 30 fractions. Patient was followed up with six monthly CT scan of thorax, abdomen
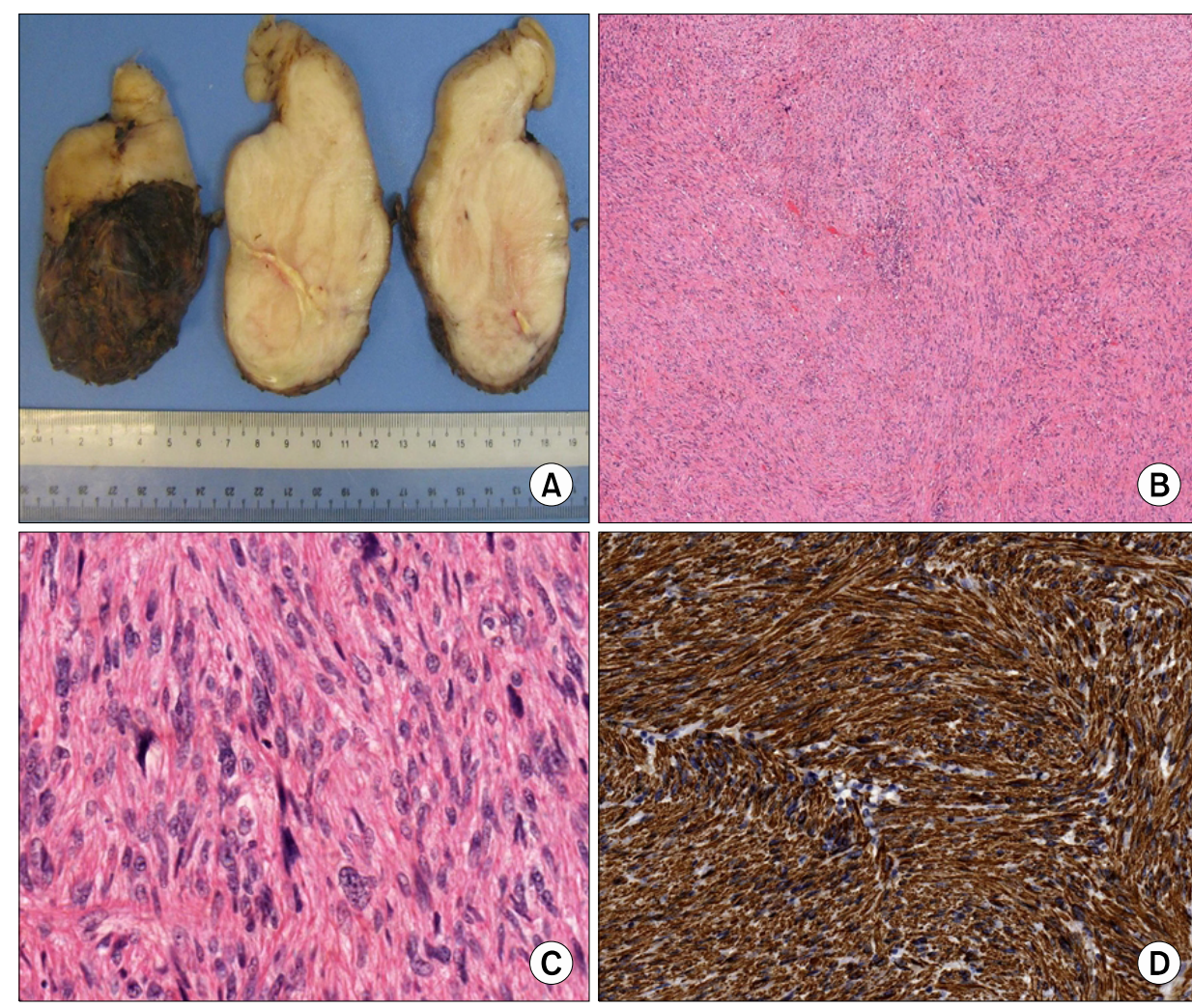

Fig. 2. Histopathological specimens of the tumor. (A) Macroscopic photos of resection specimen showing the characteristic fleshy appearance of a mesenchymal tumor. (B) intersecting fascicles of eosinophilic tumor cells (Haematoxylin and eosin stain $\times 50)$. (C) Areas show marked nuclear atypia with mitoses (Haematoxylin and eosin $\times 200$ ). (D) The tumor cells show strong desmin positivity (Desmin stain $\times 200$ ). 
and pelvis for two years and thereafter yearly ultrasound of the hepatobiliary system along with liver function tests. She remained disease free for 4 years. She died following a fall at home resulting in subarachnoid haemorrhage.

\section{DISCUSSION}

Leiomyosarcoma is a malignant tumour of mesenchymal origin that develops from the smooth muscle cells of the tunica media. Since the first ever official scientific report on IVC LMs was published in $1827,{ }^{6}$ there have been less than 400 published reports. ${ }^{5}$ Though half of all venous sarcomas arise from the $\mathrm{IVC}^{7}$ the actual incidence reported in clinical practice is few and far between. Our case report describes an octogenarian patient managed by a hepatobiliary surgeon for an indeterminate mass originating from gallbladder bed with an eventual post-operative diagnosis of IVC LM.

IVC LM is classified based on its location with respect to its adjacent organs as defined by Kulaylat et al. ${ }^{8}$ The following three levels are proposed: (a) Level 1 - infrarenal IVC, (b) Level 2 - between renal vein inferiorly and supra-hepatic veins cranially and (c) Level 3 - from the right atrium to the supra-hepatic veins. Our patient had a Level 2 IVC LM which is found to be the most common subtype in literature $(46 \%){ }^{8}$ Level 2 IVC is technically challenging to manage due to its close proximity to hepatic hilum, duodenum, right adrenal gland and short hepatic veins connecting liver to inferior vena cava. Surgical management of Level 2 IVC LM needs skill set for liver mobilization, duodenal kocherization, control of hepatic hilum and proximal and distal control of retrohepatic vena cava. This operative field is mostly the realm of hepatobiliary surgery and hence it is apt that patient is managed by specialist hepatobiliary surgery team as with our patient.

Not unlike many studies, the patient in the case report is also female. Female preponderance of up to five times is reported. ${ }^{9}$ However, the occurrence of this tumour in octogenarian is uncommon. The reported incidences of IVC LM is in the $5^{\text {th }}$ to $6^{\text {th }}$ decade ${ }^{10}$ and the oldest age reported from recent studies is that of an 81 year old lady ${ }^{6}$ who eventually succumbed to the disease in 9 months.

The usual clinical presentation is largely non-specific which results in patient's late presentation and Mingoli et al. ${ }^{11}$ has shown than more than $50 \%$ of cases were diagnosed at autopsy. We postulate that this tumour doesn't cause typical malignancy related symptoms and hence diagnosis is not made unless liberal imaging policy is employed by clinicians. Deep location precludes a clinical diagnosis by palpation unless in advanced stages. We believe that with liberal use of abdominal imaging, the detection rates of IVC LMs are higher. CT scan is the initial imaging modality and it reveals the characteristic lobulation, heterogenicity and sometimes central necrosis and hemorrhages. Our patient did not exhibit central necrosis but imaging showed lobulation with expanding and pushing growth pattern rather than infiltrative and invading pattern. Our patient also had calcification within the growth and this is also reported by others; albeit uncommonly. ${ }^{12}$

Surgical resection is the only cure and the role of chemotherapy or radiotherapy is not defined. In fact, $49.4 \%$ of patients have a 5-year overall survival after surgical resection, though disease-free survival drops to $31.4 \%$ with close to half the cohort recurring in 30 months. ${ }^{13}$ In general, one centimeter histologic negative margins are advocated; albeit, any form of R0 resection is acceptable. The effect of overall survival and disease free survival in patients with R0 or R1 resection is equivocal but it is clear that R2 resections have uniformly dismal prognosis with $0 \%$ survival in 5 years. ${ }^{14}$ Therefore, in pursuit of achieving negative margins, extensive multivisceral and vascular resections with reconstructions are reported to be performed. ${ }^{15}$ Due to the need for complex multivisceral resections, IVC LM management needs diverse surgical skill set including urology, vascular, general oncology and hepatobiliary surgery. Ours is the first report advocating the role of hepatobiliary surgeon in management of this complex disease. While surgical treatment is established as gold standard, adjuvant therapy's place in the management of IVC LM is less clear. ${ }^{16}$ In fact, only one study showed benefit when RT was employed resulting in lower recurrence rates. ${ }^{14}$ Perhaps, prospective studies are required to ascertain the value of adjuvant therapy in this rare neoplasm.

\section{REFERENCES}

1. Kwon TW, Sung KB, Cho YP, Kim DK, Yang SM, Ro JY, et 
al. Pararenal leiomyosarcoma of the inferior vena cava. J Korean Med Sci 2003;18:355-359.

2. Hollenbeck ST, Grobmyer SR, Kent KC, Brennan MF. Surgical treatment and outcomes of patients with primary inferior vena cava leiomyosarcoma. J Am Coll Surg 2003;197:575-579.

3. Laskin WB, Fanburg-Smith JC, Burke AP, Kraszewska E, Fetsch $\mathrm{JF}$, Miettinen M. Leiomyosarcoma of the inferior vena cava: clinicopathologic study of 40 cases. Am J Surg Pathol 2010;34: 873-881.

4. Mingoli A, Cavallaro A, Sapienza P, Di Marzo L, Feldhaus RJ, Cavallari N. International registry of inferior vena cava leiomyosarcoma: analysis of a world series on 218 patients. Anticancer Res 1996;16:3201-3205.

5. Wachtel H, Gupta M, Bartlett EK, Jackson BM, Kelz RR, Karakousis GC, et al. Outcomes after resection of leiomyosarcomas of the inferior vena cava: a pooled data analysis of 377 cases. Surg Oncol 2015;24:21-27.

6. Spinelli A, Schumacher G, Benckert C, Sauer IM, Schmeding M, Glanemann M, et al. Surgical treatment of a leiomyosarcoma of the inferior vena cava involving the hepatic and renal veins confluences: technical aspects. Eur J Surg Oncol 2008;34:831835.

7. Kevorkian J, Cento DP. Leiomyosarcoma of large arteries and veins. Surgery 1973;73:390-400.

8. Kulaylat MN, Karakousis CP, Doerr RJ, Karamanoukian HL, O'Brien J, Peer R. Leiomyosarcoma of the inferior vena cava: a clinicopathologic review and report of three cases. J Surg Oncol 1997;65:205-217.

9. Dzsinich C, Gloviczki P, van Heerden JA, Nagorney DM,
Pairolero PC, Johnson CM, et al. Primary venous leiomyosarcoma: a rare but lethal disease. J Vasc Surg 1992;15:595-603.

10. Liu C, Zheng Y, Yang X, Shao J, Song X, Liu B, et al. Surgical resection of the inferior vena cava for leiomyosarcoma. Ann Vasc Surg 2010;24:822.e11-e15.

11. Mingoli A, Feldhaus RJ, Cavallaro A, Stipa S. Leiomyosarcoma of the inferior vena cava: analysis and search of world literature on 141 patients and report of three new cases. J Vasc Surg 1991; 14:688-699.

12. Monteagudo Cortecero J, Guirau Rubio MD, Payá Romá A. Leiomyosarcoma of the inferior vena cava: AIRP best cases in radiologic-pathologic correlation. Radiographics 2015;35:616-620.

13. Alkhalili E, Greenbaum A, Langsfeld M, Marek J, Rana MA, Glew R, et al. Leiomyosarcoma of the inferior vena cava: a case series and review of the literature. Ann Vasc Surg 2016;33:245251.

14. Hines OJ, Nelson S, Quinones-Baldrich WJ, Eilber FR. Leiomyosarcoma of the inferior vena cava: prognosis and comparison with leiomyosarcoma of other anatomic sites. Cancer 1999;85: 1077-1083.

15. Teixeira FJR Jr, do Couto Netto SD, Perina ALF, Torricelli FCM, Ragazzo Teixeira L, Zerati AE, et al. Leiomyosarcoma of the inferior vena cava: survival rate following radical resection. Oncol Lett 2017;14:3909-3916.

16. Ito $\mathrm{H}$, Hornick JL, Bertagnolli MM, George S, Morgan JA, Baldini EH, et al. Leiomyosarcoma of the inferior vena cava: survival after aggressive management. Ann Surg Oncol 2007;14: 3534-3541. 